Homology, Homotopy and Applications, vol.14(1), 2012, pp.65-78

\title{
JACOBI-ZARISKI EXACT SEQUENCE FOR HOCHSCHILD HOMOLOGY AND CYCLIC (CO)HOMOLOGY
}

\author{
ATABEY KAYGUN \\ (communicated by Claude Cibils)
}

\begin{abstract}
We prove that for an inclusion of unital associative but not necessarily commutative $\mathbb{k}$-algebras $\mathcal{B} \subseteq \mathcal{A}$ we have long exact sequences in Hochschild homology and cyclic (co)homology akin to the Jacobi-Zariski sequence in André-Quillen homology, provided that the quotient $\mathcal{B}$-module $\mathcal{A} / \mathcal{B}$ is flat. We also prove that for an arbitrary r-flat morphism $\varphi: \mathcal{B} \rightarrow \mathcal{A}$ with an $\mathrm{H}$-unital kernel, one can express the Wodzicki excision sequence and our Jacobi-Zariski sequence in Hochschild homology and cyclic (co)homology as a single long exact sequence.
\end{abstract}

\section{Introduction}

Let $\mathbb{k}$ be a ground field. Assume we have an inclusion of associative commutative unital $\mathbb{k}$-algebras $B \subseteq A$. Then for any $A$-bimodule $N$, one obtains a long exact sequence in André-Quillen homology [12, Thm. 5.1]

$$
\cdots \rightarrow D_{n+1}(A \mid B ; N) \rightarrow D_{n}(B \mid \mathbb{k} ; N) \rightarrow D_{n}(A \mid \mathbb{k} ; N) \rightarrow D_{n}(A \mid B ; N) \rightarrow \cdots,
$$

which is often referred to as the Jacobi-Zariski long exact sequence $[\mathbf{8}, \S 3.5]$. In this paper we show that there are analogous long exact sequences for ordinary (co)homology, Hochschild homology and cyclic (co)homology of $\mathbb{k}$-algebras of the form (written here for Hochschild homology)

$$
\cdots \rightarrow H H_{n+1}(\mathcal{A} \mid \mathcal{B}) \rightarrow H H_{n}(\mathcal{B} \mid \mathbb{k}) \rightarrow H H_{n}(\mathcal{A} \mid \mathbb{k}) \rightarrow H H_{n}(\mathcal{A} \mid \mathcal{B}) \rightarrow \cdots
$$

We prove the existence under the condition that we have a unital associative (not necessarily commutative) algebra $\mathcal{A}$ and a unital subalgebra $\mathcal{B}$ such that the quotient $\mathcal{B}$-module $\mathcal{A} / \mathcal{B}$ is flat. In the sequel, such inclusions $\mathcal{B} \subseteq \mathcal{A}$ of unital $\mathbb{k}$-algebras are called reduced-flat (r-flat in short) extensions. The condition of $\mathrm{r}$-flatness is slightly more restrictive than $\mathcal{A}$ being flat over $\mathcal{B}$, but there are plenty of relevant examples. (See Subsection 1.5.)

There are similar long exact sequences in the literature for other cohomology theories of $\mathbb{k}$-algebras. The relevant sequence we consider here is the Wodzicki excision

Received June 1, 2011, revised September 24, 2011, October 2, 2011, October 27, 2011; published on May 7, 2012.

2000 Mathematics Subject Classification: 19D55, 18G25, 16W70, 18G40.

Key words and phrases: Jacobi-Zariski sequence, excision, Hochschild homology, cyclic cohomology. Article available at http://intlpress.com/HHA/v14/n1/a4 and doi:10.4310/HHA.2012.v14.n1.a4 Copyright (C) 2012, International Press. Permission to copy for private use granted. 
sequence [13] for Hochschild homology and cyclic (co)homology (written here for Hochschild homology)

$$
\cdots \rightarrow H H_{n}(\mathcal{I}) \rightarrow H H_{n}(\mathcal{B}) \rightarrow H H_{n}(\mathcal{A}) \rightarrow H H_{n-1}(\mathcal{I}) \cdots
$$

for an epimorphism $\pi: \mathcal{B} \rightarrow \mathcal{A}$ of unital $\mathbb{k}$-algebras with an H-unital kernel $\mathcal{I}:=$ $\operatorname{ker}(\pi)$ (Subsection 1.3). The Wodzicki excision sequence characterizes homotopy cofiber of the morphism of differential graded $\mathbb{k}$-modules $\pi_{*}: \mathrm{CH}_{*}(\mathcal{B}) \rightarrow \mathrm{CH}_{*}(\mathcal{A})$ induced by $\pi$ as the suspended Hochschild complex $\Sigma \mathrm{CH}_{*}(\mathcal{I})$ of the ideal $\mathcal{I}$. Our Jacobi-Zariski sequence, on the other hand, characterizes the same homotopy cofiber as the relative Hochschild chain complex $\mathrm{CH}_{*}(\mathcal{A} \mid \mathcal{B})$ (relative à la Hochschild $[\mathbf{6}]$ ) for a monomorphism $\mathcal{B} \rightarrow \mathcal{A}$ of $\mathbb{k}$-algebras.

Now, assume $\varphi: \mathcal{B} \rightarrow \mathcal{A}$ is an arbitrary morphism of unital $\mathbb{k}$-algebras such that $\mathcal{I}:=\operatorname{ker}(\varphi)$ is $\mathrm{H}$-unital and the quotient $\mathcal{B}$-module $\mathcal{A} / \operatorname{im}(\varphi)$ is flat. Under these conditions, we show in Theorem 4.3 (written here for Hochschild chain complexes) that homotopy cofiber $\mathrm{CH}_{*}(\mathcal{A}, \mathcal{B})$ of the morphism $\varphi_{*}: \mathrm{CH}_{*}(\mathcal{B}) \rightarrow \mathrm{CH}_{*}(\mathcal{A})$ induced by $\varphi$ fits into a homotopy cofibration sequence of the form

$$
\Sigma \mathrm{CH}_{*}(\mathcal{I}) \rightarrow \mathrm{CH}_{*}(\mathcal{A}, \mathcal{B}) \rightarrow \mathrm{CH}_{*}(\mathcal{A} \mid \mathcal{B}),
$$

which gives us an appropriate long exact sequence. As one can immediately see, we get Wodzicki's characterization of the homotopy cofiber when $\varphi$ is an epimorphism and our Jacobi-Zariski characterization when $\varphi$ is a monomorphism.

\section{Overview}

In Section 1 we review some standard constructions and facts that we are going to need in the course of proving our main result, mostly in order to establish notation. Then in Section 2 we prove the existence of the Jacobi-Zariski long exact sequence for ordinary homology of algebras. In Section 3 we gradually develop the same result for cohomology under certain restrictions on the dimension of the algebra, and then we remove those restrictions and place them on the coefficient modules. Finally, in Section 4, we prove the existence of the Jacobi-Zariski long exact sequence for Hochschild homology and cyclic (co)homology of associative unital algebras, and then construct a homotopy cofibration sequence extending both the Jacobi-Zariski sequence and Wodzicki excision sequence in Hochschild homology and cyclic (co)homology.

\section{Standing assumptions and conventions}

We use $\mathbb{k}$ to denote our ground field. We make no assumptions on the characteristic of $\mathbb{k}$. All unadorned tensor products $\otimes$ are assumed to be over $\mathbb{k}$. We will use $\bigoplus_{i} X_{i}$ and $\sum_{i} X_{i}$ to denote respectively the external and the internal sum of a collection of subspaces $\left\{X_{i}\right\}_{i \in I}$ of a $\mathbb{k}$-vector space $V$. The $\mathbb{k}$-algebras we consider are all unital and associative but not necessarily commutative. We make no assumptions on the $\mathbb{k}$-dimensions of these algebras unless it is otherwise explicitly stated. We will use $\mathcal{A}^{e}$ to denote the enveloping algebra $\mathcal{A} \otimes \mathcal{A}^{o p}$ of an associative unital algebra $\mathcal{A}$. We will use the term parity to denote the type of an $\mathcal{A}$-module: whether it is a left module or a right module. We use the notation $\Sigma C_{*}$ for the suspension of a differential $\mathbb{Z}$-graded $\mathbb{k}$-module $\left(C_{*}, d_{*}^{C}\right)$, where

$$
(\Sigma C)_{n}=C_{n-1} \quad \text { and } \quad d_{n}^{\Sigma C}=-d_{n-1}^{C}
$$

for every $n \in \mathbb{Z}$. 


\section{Acknowledgements}

We are grateful to the referee for pointing out the reference $[\mathbf{2}]$ which allowed us to connect H-unital ideals of Wodzicki [13] and homological epimorphisms of [3]. We would like to thank the referee also for pointing out the references $[\mathbf{1}, \mathbf{5}]$, and $[\mathbf{1 0}]$ which allowed us to better contextualize Conjecture 4.4.

\section{Preliminaries}

\subsection{Relative (co)homology}

A monomorphism $f: X \rightarrow Y$ of $\mathcal{A}$-modules is called an $(\mathcal{A}, \mathcal{B})$-monomorphism if $f$ is an monomorphism of $\mathcal{A}$-modules and a split monomorphism of $\mathcal{B}$-modules. We define $(\mathcal{A}, \mathcal{B})$-epimorphisms similarly. A short exact sequence of $\mathcal{A}$-modules $0 \rightarrow$ $X \stackrel{i}{\longrightarrow} Y \stackrel{p}{\longrightarrow} Z \rightarrow 0$ is called an $(\mathcal{A}, \mathcal{B})$-exact sequence if $i$ is an $(\mathcal{A}, \mathcal{B})$-monomorphism and $p$ is an $(\mathcal{A}, \mathcal{B})$-epimorphism.

An $\mathcal{A}$-module $P$ is called an $(\mathcal{A}, \mathcal{B})$-projective module if for any $(\mathcal{A}, \mathcal{B})$-epimorphism $f: X \rightarrow Y$ and a morphism of $\mathcal{A}$-modules $g: P \rightarrow Y$ there exists an $\mathcal{A}$-module morphism $g^{\prime}: P \rightarrow X$ which satisfies $g=f \circ g^{\prime}$

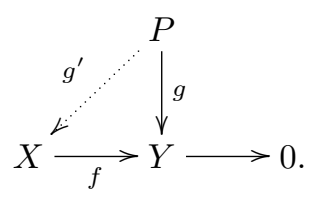

$(\mathcal{A}, \mathcal{B})$-injective modules are defined similarly. Also, a module $T$ is called $(\mathcal{A}, \mathcal{B})$-flat if for every $(\mathcal{A}, \mathcal{B})$-short exact sequence $0 \rightarrow X \rightarrow X^{\prime} \rightarrow X^{\prime \prime} \rightarrow 0$ the induced sequence of $\mathbb{k}$-modules

$$
0 \rightarrow X \otimes_{\mathcal{A}} T \rightarrow X^{\prime} \otimes_{\mathcal{A}} T \rightarrow X^{\prime \prime} \otimes_{\mathcal{A}} T \rightarrow 0
$$

is exact. Note that every $\mathcal{A}$-projective (resp. $\mathcal{A}$-flat or $\mathcal{A}$-injective) module is also $(\mathcal{A}, \mathcal{B})$-projective (resp. $(\mathcal{A}, \mathcal{B})$-flat or $(\mathcal{A}, \mathcal{B})$-injective) for any unital subalgebra $\mathcal{B} \subseteq \mathcal{A}$.

\subsection{The bar complex}

The bar complex of a (unital) associative algebra $\mathcal{A}$ is the graded $\mathbb{k}$-module

$$
\mathrm{CB}_{*}(\mathcal{A}):=\bigoplus_{n \geqslant 0} \mathcal{A}^{\otimes n+2}
$$

together with the differentials $d_{n}^{\mathrm{CB}}: \mathrm{CB}_{n}(\mathcal{A}) \rightarrow \mathrm{CB}_{n-1}(\mathcal{A})$ which are defined by

$$
d_{n}^{\mathrm{CB}}\left(a_{0} \otimes \cdots \otimes a_{n+1}\right)=\sum_{j=0}^{n}(-1)^{j}\left(\cdots \otimes a_{j} a_{j+1} \otimes \cdots\right)
$$

for any $n \geqslant 1$ and $a_{0} \otimes \cdots \otimes a_{n} \in \mathrm{CB}_{n}(\mathcal{A})$. The complex

$$
\mathrm{CB}_{*}(X ; \mathcal{A} ; Y):=X \otimes_{\mathcal{A}} \mathrm{CB}_{*}(\mathcal{A}) \otimes_{\mathcal{A}} Y
$$

is called the two-sided (homological) bar complex of a pair $(X, Y)$ of $\mathcal{A}$-modules of opposite parity. The cohomological counterpart $\mathrm{CB}^{*}(X ; \mathcal{A} ; Z)$ for a pair of right 
$\mathcal{A}$-modules $(X, Z)$ is defined as

$$
\mathrm{CB}^{*}(X ; \mathcal{A} ; Z):=\operatorname{Hom}_{\mathcal{A}}\left(\mathrm{CB}_{*}(X ; \mathcal{A} ; \mathcal{A}), Z\right) .
$$

The corresponding bar complex for left modules is defined similarly using $\mathrm{CB}_{*}(\mathcal{A} ; \mathcal{A} ; X)$. Since the two-sided bar complex $\mathrm{CB}_{*}(\mathcal{A})$ is an $\mathcal{A}^{e}$-projective resolution of the $\mathcal{A}$-bimodule $\mathcal{A}$, the homology of the complex $\mathrm{CB}_{*}(X ; \mathcal{A} ; Y)$ yields the Tor-groups $\operatorname{Tor}_{*}^{\mathcal{A}}(X, Y)$ for the pair $(X, Y)$. Similarly, the Ext-groups $\operatorname{Ext}_{\mathcal{A}}^{*}(X, Z)$ for the pair $(X, Z)$ come from the cohomological variant of the two-sided bar complex $\mathrm{CB}^{*}(X ; \mathcal{A} ; Z)$.

To be technically correct, since our tensor products $\otimes$ are taken over $\mathbb{k}$, the bar complexes we defined should be referred as the relative bar complexes (relative to the base field) denoted by $\mathrm{CB}_{*}(X ; \mathcal{A} \mid \mathbb{k} ; Y)$ or $\mathrm{CB}^{*}(X ; \mathcal{A} \mid \mathbb{k} ; Z)$. However, since $\mathbb{k}$ is a semisimple subalgebra of $\mathcal{A}$, the relative (co)homology and the absolute (co)homology agree (cf. [7, Prop. 2.5]). See also [4, Thm. 1.2].

Now, we define the relative two-sided bar complexes

$$
\mathrm{CB}_{*}(X ; \mathcal{A} \mid \mathcal{B} ; Y) \text { and } \mathrm{CB}^{*}(X ; \mathcal{A} \mid \mathcal{B} ; Z)
$$

similarly for $\mathcal{A}$-modules of the correct parity $X, Y$ and $Z$, where we replace the tensor product $\otimes$ over $\mathbb{k}$ with the tensor product $\otimes_{\mathcal{B}}$ over a unital subalgebra $\mathcal{B}$. Since $\mathcal{A} \otimes_{\mathcal{B}} \mathcal{A}$ is $(\mathcal{A}, \mathcal{B})$-projective by [6, Lem. 2, p. 248], we see that for any right $\mathcal{A}$-module $X$, the module $X \otimes_{\mathcal{B}} \mathcal{A}$ is a $(\mathcal{A}, \mathcal{B})$-projective module. Then the relative complexes $\mathrm{CB}_{*}(X ; \mathcal{A} \mid \mathcal{B} ; Y)$ and $\mathrm{CB}^{*}(X ; \mathcal{A} \mid \mathcal{B} ; Z)$ yield respectively the relative torsion groups $\operatorname{Tor}_{*}^{(\mathcal{A} \mid \mathcal{B})}(X, Y)$ and the relative extension groups $\operatorname{Ext}_{(\mathcal{A} \mid \mathcal{B})}^{*}(X, Z)$.

\subsection{H-unital ideals}

A not necessarily unital $\mathbb{k}$-algebra $\mathcal{I}$ is called $\mathrm{H}$-unital if the bar complex $\mathrm{CB}_{*}(\mathcal{I})$ of $\mathcal{I}$ is a resolution of $\mathcal{I}$ viewed as a $\mathcal{I}$-bimodule. One can immediately see that any unital algebra is H-unital.

Our definition of H-unitality differs from Wodzicki's original definition given in [13], but is still equivalent. Wodzicki defines another complex $\mathrm{CB}_{*}^{\prime}(\mathcal{I})$ (he uses the notation $\left.B_{*}(\mathcal{I})\right)$

$$
\mathrm{CB}_{*}^{\prime}(\mathcal{I})=\bigoplus_{n \geqslant 0} \mathcal{I}^{\otimes n+1}
$$

together with the differentials

$$
d_{n}\left(x_{0} \otimes \cdots \otimes x_{n}\right)=\sum_{j=0}^{n-1}(-1)^{j}\left(\cdots \otimes x_{j} x_{j+1} \otimes \cdots\right) .
$$

$\mathrm{CB}_{0}^{\prime}(\mathcal{I})$ is the differential graded $\mathbb{k}$-module $\mathrm{CB}_{*}(\mathcal{I})$ suspended once and augmented by $\mathcal{I}$. The fact that $\mathrm{CB}_{*}(\mathcal{I})$ is a resolution of $\mathcal{I}$ is equivalent to the fact that $\mathrm{CB}_{*}^{\prime}(\mathcal{I})$ is acyclic. Because $\mathbb{k}$ is a field and every $\mathbb{k}$-module is free, and therefore flat, the acyclicity of $\mathrm{CB}_{*}^{\prime}(\mathcal{I})$ is equivalent to Wodzicki's definition of H-unitality (cf. [13, p. 592].) 


\subsection{The Hochschild complex}

The Hochschild complex of $\mathcal{A}$ with coefficients in a $\mathcal{A}$-bimodule $M$ is the graded $\mathbb{k}$-module

$$
\mathrm{CH}_{*}(\mathcal{A}, M):=\bigoplus_{n \geqslant 0} M \otimes \mathcal{A}^{\otimes n}
$$

together with the differentials (traditionally denoted by $b$ )

$$
\begin{gathered}
b_{n}: \mathrm{CH}_{n}(\mathcal{A}, M) \rightarrow \mathrm{CH}_{n-1}(\mathcal{A}, M), \\
b_{n}\left(m \otimes a_{1} \otimes \cdots a_{n}\right)=\left(m a_{1} \otimes a_{2} \otimes \cdots \otimes a_{n}\right)+\sum_{j=1}^{n-1}(-1)^{j}\left(m \otimes \cdots \otimes a_{j} a_{j+1} \otimes \cdots\right) \\
+(-1)^{n}\left(a_{n} m \otimes a_{1} \otimes \cdots \otimes a_{n-1}\right)
\end{gathered}
$$

defined for $n \geqslant 1$ and $m \otimes a_{1} \otimes \cdots \otimes a_{n} \in \mathrm{CH}_{n}(\mathcal{A}, M)$. One can define the relative Hochschild complex $\mathrm{CH}_{*}(\mathcal{A} \mid \mathcal{B}, M)$ similarly, where we write the tensor products over $\mathcal{B}$ instead of $\mathbb{k}$.

For every $\mathcal{A}$-bimodule $M$ the Hochschild complex $\mathrm{CH}_{*}(\mathcal{A}, M)$ can also be constructed as

$$
\mathrm{CH}_{*}(\mathcal{A}, M)=M \otimes_{\mathcal{A}^{e}} \mathrm{CB}_{*}(\mathcal{A}),
$$

where $\mathcal{A}^{e}=\mathcal{A} \otimes \mathcal{A}^{o p}$ is the enveloping algebra of $\mathcal{A}$. Since the bar complex $\mathrm{CB}_{*}(\mathcal{A})$ is a projective resolution of $\mathcal{A}$ viewed as a $\mathcal{A}$-bimodule, the Hochschild homology groups we defined using the Hochschild complex are $\operatorname{Tor}_{n}^{\mathcal{A}^{e}}(\mathcal{A}, M)$ [8, Prop. 1.1.13]. One can similarly define the Hochschild cohomology groups $H H^{n}(\mathcal{A}, M)$ as the extension groups $\operatorname{Ext}_{\mathcal{A}^{e}}^{n}(\mathcal{A}, M)$, which can also be computed as the cohomology of the Hochschild cochain complex $\mathrm{CH}^{*}(\mathcal{A}, M):=\operatorname{Hom}_{\mathcal{A}^{e}}\left(\mathrm{CB}_{*}(\mathcal{A}), M\right)[\mathbf{8}$, Def. 1.5.1].

We will use the notation $H H_{*}(\mathcal{A})$ and $H H^{*}(\mathcal{A})$ to denote respectively $H H_{*}(\mathcal{A}, \mathcal{A})$ and $H H^{*}(\mathcal{A}, \mathcal{A})$ for a (unital) $\mathbb{k}$-algebra $\mathcal{A}$.

\subsection{Flat and r-flat extensions}

Assume $\mathcal{B} \subseteq \mathcal{A}$ is a unital subalgebra. This makes $\mathcal{A}$ into a $\mathcal{B}$-bimodule. Now, consider the following short exact sequence of $\mathcal{B}$-modules

$$
0 \rightarrow \mathcal{B} \rightarrow \mathcal{A} \rightarrow \mathcal{A} / \mathcal{B} \rightarrow 0
$$

Since $\mathcal{B}$ is flat over itself, using the short exact sequence above for every $\mathcal{B}$-module $Y$ we get an exact sequence of $\mathbb{k}$-modules of the form

$$
0 \rightarrow \operatorname{Tor}_{1}^{\mathcal{B}}(\mathcal{A}, Y) \rightarrow \operatorname{Tor}_{1}^{\mathcal{B}}(\mathcal{A} / \mathcal{B}, Y) \rightarrow \mathcal{B} \otimes_{\mathcal{B}} Y \rightarrow \mathcal{A} \otimes_{\mathcal{B}} Y \rightarrow(\mathcal{A} / \mathcal{B}) \otimes_{\mathcal{B}} Y \rightarrow 0
$$

and an isomorphism of $\mathbb{k}$-modules $\operatorname{Tor}_{n}^{\mathcal{B}}(\mathcal{A}, Y) \cong \operatorname{Tor}_{n}^{\mathcal{B}}(\mathcal{A} / \mathcal{B}, Y)$ for every $n \geqslant 2$. This means the flat dimension (sometimes referred as the weak dimension) of $\mathcal{A} / \mathcal{B}$ is at most 1 when $\mathcal{A}$ is a flat $\mathcal{B}$-module. On the other hand, when the quotient $\mathcal{A} / \mathcal{B}$ is a flat $\mathcal{B}$-module the $\mathcal{B}$-module $\mathcal{A}$ must also be flat.

Definition 1.1. An inclusion of unital $\mathbb{k}$-algebras $\mathcal{B} \subseteq \mathcal{A}$ is called a flat extension if $\mathcal{A}$ viewed as a $\mathcal{B}$-bimodule is flat. An inclusion of unital $\mathbb{k}$-algebras $\mathcal{B} \subseteq \mathcal{A}$ is called a $r$-flat extension if the quotient $\mathcal{B}$-bimodule $\mathcal{A} / \mathcal{B}$ is $\mathcal{B}$-flat. A morphism of unital $\mathbb{k}$-algebras $\varphi: \mathcal{B} \rightarrow \mathcal{A}$ is called flat (resp. r-flat) if $\operatorname{im}(\varphi) \subseteq \mathcal{A}$ is a flat (resp. r-flat) extension. 
One can easily see that every r-flat extension is a flat extension. Conversely, if $\mathcal{A}$ is a flat extension over $\mathcal{B}$ which is also augmented, i.e., we have a unital algebra morphism $\varepsilon: \mathcal{A} \rightarrow \mathcal{B}$ splitting the inclusion of algebras $\mathcal{B} \rightarrow \mathcal{A}$, then it is also a r-flat extension. In other words, for augmented extensions flatness and r-flatness are equivalent.

Example 1.2. The polynomial algebras $\mathcal{B}\left[x_{1}, \ldots, x_{n}\right]$ with commuting indeterminates and the polynomial algebras $\mathcal{B}\left\{x_{1}, \ldots, x_{n}\right\}$ with non-commuting indeterminates are all $\mathrm{r}$-flat extensions of a unital $\mathbb{k}$-algebra $\mathcal{B}$. In general, if $\mathcal{G}$ is a monoid then the group algebra $\mathcal{B}[\mathcal{G}]$ of $\mathcal{G}$ over $\mathcal{B}$ gives us a r-flat extension $\mathcal{B} \subseteq \mathcal{B}[\mathcal{G}]$.

\section{The Jacobi-Zariski sequence for torsion groups}

Proposition 2.1. Let $\mathcal{I}$ be an $\mathrm{H}$-unital ideal of a unital algebra $\mathcal{B}$. Assume $X$ and $Y$ are $\mathcal{B} / \mathcal{I}$-modules of opposite parity. Then one has natural isomorphisms of the form

$$
\operatorname{Tor}_{p}^{\mathcal{B}}(X, Y) \cong \operatorname{Tor}_{p}^{\mathcal{B} / \mathcal{I}}(X, Y)
$$

for every $p \geqslant 0$.

Proof. We begin by defining an auxiliary differential graded $\mathbb{k}$-module $I_{*}$. We augment $\mathrm{CB}_{*}^{\prime}(\mathcal{I})$ with $\mathbb{k}$ as follows:

$$
\mathbb{k} \stackrel{0}{\leftarrow} \mathcal{I} \leftarrow \mathcal{I}^{\otimes 2} \leftarrow \mathcal{I}^{\otimes 3} \leftarrow \cdots .
$$

Since $\mathcal{I}$ is H-unital, the homology is concentrated at degree 0 where $H_{0}\left(I_{*}\right)=\mathbb{k}$ and $H_{q}\left(I_{*}\right)=0$ for every $q>0$. Then the product $X \otimes I_{*} \otimes Y$ is a differential graded $\mathbb{k}$-submodule of $\mathrm{CB}_{*}(X ; \mathcal{B} ; Y)$ since action of $\mathcal{I}$ on $X$ and $Y$ are by 0 . Now, we define an increasing filtration of differential graded $\mathbb{k}$-submodules $F_{*}^{p} \subseteq F_{*}^{p+1}$ of $\mathrm{CB}_{*}(X ; \mathcal{B} ; Y)$, where for $0 \leqslant p \leqslant n$ we let

$$
F_{n}^{p}:=\sum_{n_{0}+\cdots+n_{p}=n-p} X \otimes \mathcal{I}^{\otimes n_{0}} \otimes \mathcal{B} \otimes \mathcal{I}^{\otimes n_{1}} \otimes \cdots \otimes \mathcal{B} \otimes \mathcal{I}^{\otimes n_{p}} \otimes Y
$$

and allow $n_{i}=0$. Then we define $F_{n}^{p}=F_{n}^{p+1}$ for every $p \geqslant n$ and let $F_{*}^{-1}:=0$. Note that $F_{*}^{0}=X \otimes I_{*} \otimes Y$ and $\bigcup_{p} F_{*}^{p}=\mathrm{CB}_{*}(X ; \mathcal{B} ; Y)$. In the associated spectral sequence of the filtration we get

$$
E_{0, q}^{1}=H_{q}\left(F_{*}^{0} / F_{*}^{-1}\right)=H_{q}\left(X \otimes I_{*} \otimes Y\right) \cong X \otimes H_{q}\left(I_{*}\right) \otimes Y=0=E_{0, q}^{\infty}
$$

for every $q>0$ since $\mathcal{I}$ is H-unital and $\otimes$ is an exact bifunctor. We also see that for $q<0$

$$
E_{p, q}^{0}=F_{p+q}^{p} / F_{p+q}^{p-1}=0=E_{p, q}^{\infty}
$$

because $F_{n}^{p}=F_{n}^{p+1}$ for every $p \geqslant n$. Moreover, for any $p>0$ and for any $q \geqslant 0$,

$$
\begin{aligned}
E_{p, q}^{0}=F_{p+q}^{p} / F_{p+q}^{p-1} & \cong \bigoplus_{n_{0}+\cdots+n_{p}=q} X \otimes \mathcal{I}^{\otimes n_{0}} \otimes(\mathcal{B} / \mathcal{I}) \otimes \mathcal{I}^{\otimes n_{1}} \otimes \cdots \otimes(\mathcal{B} / \mathcal{I}) \otimes \mathcal{I}^{\otimes n_{p}} \otimes Y .
\end{aligned}
$$

Again, since the action of $\mathcal{I}$ on $X, Y$ and $\mathcal{B} / \mathcal{I}$ are by 0 , the quotient $F_{*}^{p} / F_{*}^{p-1}$ can 
be written as a product of differential graded modules

$$
\Sigma^{p}\left(F_{*}^{p} / F_{*}^{p-1}\right) \cong X \otimes \underbrace{I_{*} \otimes(\mathcal{B} / \mathcal{I}) \otimes \cdots \otimes I_{*} \otimes(\mathcal{B} / \mathcal{I})}_{p \text {-times }} \otimes I_{*} \otimes Y .
$$

Our spectral sequence collapses because the homology of $I_{*}$ is concentrated at degree 0 . So, we get $E_{p, q}^{1}=0=E_{p, q}^{\infty}$ for $q \neq 0$ and for $p \geqslant 0$ we get

$$
E_{p, 0}^{1}=H_{p}\left(F_{*}^{p} / F_{*}^{p-1}\right)=X \otimes(\mathcal{B} / \mathcal{I})^{\otimes p} \otimes Y,
$$

which means $E_{*, 0}^{1}=\mathrm{CB}_{*}(X ; \mathcal{B} / \mathcal{I} ; Y)$. Hence we get $E_{p, 0}^{2}=E_{p, 0}^{\infty}=\operatorname{Tor}_{p}^{\mathcal{B} / \mathcal{I}}(X, Y)$. Our spectral sequence converges to the homology of $\mathrm{CB}_{*}(X ; \mathcal{B} ; Y)$ which is $\operatorname{Tor}_{*}^{\mathcal{B}}(X, Y)$. The result follows.

Remark 2.2. An epimorphism of algebras $\varphi: \mathcal{B} \rightarrow \mathcal{A}$ is called a homological epimorphism if the induced morphisms $\operatorname{Ext}_{\mathcal{A}}^{*}(X, Y) \rightarrow \operatorname{Ext}_{\mathcal{B}}^{*}(X, Y)$ is an isomorphism for every finitely generated $\mathcal{A}$-modules $X$ and $Y[\mathbf{3}]$. If $\mathcal{B}$ is finite dimensional and we confine ourselves with finitely generated modules, then one has isomorphisms of the form (2.1) if and only if one has the same isomorphisms for Ext-groups. In other words, an epimorphism $\varphi: \mathcal{B} \rightarrow \mathcal{A}$ between two finite dimensional $\mathbb{k}$-algebras is a homology epimorphism when $\operatorname{ker}(\varphi)$ is H-unital because of Proposition 2.1. We would like to reiterate here again that we make no assumptions on the $\mathbb{k}$-dimensions of the algebras we work with, neither do we assume that our modules are finitely generated.

Theorem 2.3. Assume $\mathcal{B}$ is a unital $\mathbb{k}$-algebra and let $\mathcal{B} \subseteq \mathcal{A}$ be a r-flat extension. Then we have a long exact sequence of the form

$$
\cdots \rightarrow \operatorname{Tor}_{p+1}^{(\mathcal{A} \mid \mathcal{B})}(X, Y) \rightarrow \operatorname{Tor}_{p}^{\mathcal{B}}(X, Y) \rightarrow \operatorname{Tor}_{p}^{\mathcal{A}}(X, Y) \rightarrow \operatorname{Tor}_{p}^{(\mathcal{A} \mid \mathcal{B})}(X, Y) \rightarrow \cdots
$$

for any right $\mathcal{A}$-module $X$ and any left $\mathcal{A}$-module $Y$, and $p \geqslant 0$.

Proof. We consider the two-sided bar complex $\mathrm{CB}_{*}(X ; \mathcal{A} ; Y)$ for a right $\mathcal{A}$-module $X$ and for a left $\mathcal{A}$-module $Y$. We consider the following increasing filtration on $\mathrm{CB}_{*}(X ; \mathcal{A} ; Y)$

$$
L_{n}^{p}=\sum_{n_{0}+\cdots+n_{p}=n-p} X \otimes \mathcal{B}^{\otimes n_{0}} \otimes \mathcal{A} \otimes \mathcal{B}^{\otimes n_{1}} \otimes \cdots \otimes \mathcal{A} \otimes \mathcal{B}^{\otimes n_{p}} \otimes Y
$$

for $p \leqslant n$, where we allow $n_{i}=0$. We take $L_{*}^{p}=0$ for $p<0$ and let $L_{n}^{p}=L_{n}^{p+1}$ for $p \geqslant n$. Note that the filtration degree comes from the number of tensor components which are equal to $\mathcal{A}$. We also see that $L_{*}^{0}=\mathrm{CB}_{*}(X ; \mathcal{B} ; Y)$ and $\operatorname{colim}_{p} L_{*}^{p}=$ $\mathrm{CB}_{*}(X ; \mathcal{A} ; Y)$. Then it is clear that $L_{*}^{0} / L_{*}^{-1}=L_{*}^{0}=\mathrm{CB}_{*}(X ; \mathcal{B} ; Y)$, and for $n \geqslant p$ we have

$$
L_{n}^{p} / L_{n}^{p-1} \cong \bigoplus_{n_{0}+\cdots+n_{p}=n-p} X \otimes \mathcal{B}^{\otimes n_{0}} \otimes(\mathcal{A} / \mathcal{B}) \otimes \mathcal{B}^{\otimes n_{1}} \otimes \cdots \otimes(\mathcal{A} / \mathcal{B}) \otimes \mathcal{B}^{\otimes n_{p}} \otimes Y .
$$

In the derived category, the quotient differential graded $\mathbb{k}$-module $L_{*}^{p} / L_{*}^{p-1}$ represents an $(p+2)$-fold product

$$
X \otimes_{\mathcal{B}}^{L}(\mathcal{A} / \mathcal{B}) \otimes_{\mathcal{B}}^{L} \cdots \otimes_{\mathcal{B}}^{L}(\mathcal{A} / \mathcal{B}) \otimes_{\mathcal{B}}^{L} Y
$$

where $\otimes_{\mathcal{B}}^{L}$ denotes the left derived functor of $\otimes_{\mathcal{B}}$. We use the spectral sequence associated with this filtration employing the fact that $\mathcal{B} \subseteq \mathcal{A}$ is a r-flat extension and we 
see that the only non-zero groups are on the $p$-axis and on the $q$-axis

$$
E_{p, q}^{1}=H_{p+q}\left(L_{*}^{p} / L_{*}^{p-1}\right)= \begin{cases}\operatorname{Tor}_{q}^{\mathcal{B}}(X, Y) & \text { if } p=0 \\ X \otimes_{\mathcal{B}} \underbrace{(\mathcal{A} / \mathcal{B}) \otimes_{\mathcal{B}} \cdots \otimes_{\mathcal{B}}(\mathcal{A} / \mathcal{B})}_{p \text {-times }} \otimes_{\mathcal{B}} Y & \text { if } q=0 .\end{cases}
$$

Now, we observe that the edge $q=0$ on the $E^{1}$-page is the normalized bar complex of $X$ and $Y$ over $\mathcal{A}$ relative to $\mathcal{B}$ [9, Ch. VIII Thm. 6.1 and Ch. IX $\S \S 8-9]$. Then for $E_{p, q}^{2}$ we get

$$
E_{p, q}^{2}= \begin{cases}\operatorname{Tor}_{q}^{\mathcal{B}}(X, Y) & \text { if } p=0 \\ \operatorname{Tor}_{p}^{(\mathcal{A} \mid \mathcal{B})}(X, Y) & \text { if } q=0\end{cases}
$$

For the subsequent pages in this spectral sequence, the only relevant differentials are

$$
d_{p, 0}^{p}: \operatorname{Tor}_{p}^{(\mathcal{A} \mid \mathcal{B})}(X, Y) \rightarrow \operatorname{Tor}_{p-1}^{\mathcal{B}}(X, Y)
$$

which give us short exact sequences of the form

$$
0 \rightarrow E_{p, 0}^{\infty} \rightarrow \operatorname{Tor}_{p}^{(\mathcal{A} \mid \mathcal{B})}(X, Y) \rightarrow \operatorname{Tor}_{p-1}^{\mathcal{B}}(X, Y) \rightarrow E_{0, p-1}^{\infty} \rightarrow 0 .
$$

So, eventually in the $E^{\infty}$-page there remains only two classes of non-zero groups: one on the $p$-axis the other on the $q$-axis. The induced filtration $L^{*} \operatorname{Tor}_{*}^{\mathcal{A}}(X, Y)$ on the homology satisfies

$$
E_{p, q}^{\infty}=L^{p} \operatorname{Tor}_{p+q}^{\mathcal{A}}(X, Y) / L^{p-1} \operatorname{Tor}_{p+q}^{\mathcal{A}}(X, Y)
$$

which can be placed into a diagram of the form

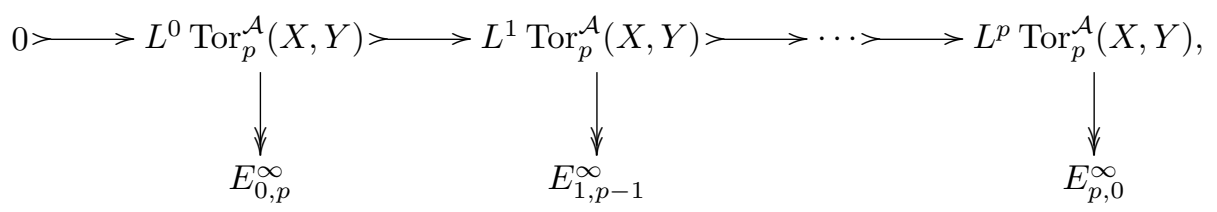

where the top row is a sequence of injections with quotients given by the appropriate groups in $E^{\infty}$. Since we only had non-zero groups on the $p$ - and $q$-axes, we get short exact sequences of the form

$$
0 \rightarrow E_{0, p}^{\infty} \rightarrow \operatorname{Tor}_{p}^{\mathcal{A}}(X, Y) \rightarrow E_{p, 0}^{\infty} \rightarrow 0 .
$$

Splicing (2.3) and (2.4) we get a long exact sequence of the form (2.2).

Corollary 2.4. Assume $\varphi: \mathcal{B} \rightarrow \mathcal{A}$ is a $\mathrm{r}$-flat morphism such that $\operatorname{ker}(\varphi)$ is an $\mathrm{H}$-unital ideal of $\mathcal{B}$. Then there is a long exact sequence of the form (2.2) for any right $\mathcal{A}$-module $X$ and any left $\mathcal{A}$-module $Y$.

Proof. There is an overload of notations here. $\operatorname{Tor}^{(\mathcal{A} \mid i m(\varphi))}$ refers to the relative Tor with $\operatorname{im}(\varphi)$ is a subalgebra of $\mathcal{A}$. On the other hand $\operatorname{Tor}^{(\mathcal{A} \mid \mathcal{B})}$ refers to relative Tor using $\varphi: \mathcal{B} \rightarrow \mathcal{A}$ which, by definition, is constructed using the image of $\varphi$. In other words, if $\mathcal{A}$ is viewed as a $\mathcal{B}$-module via a morphism $\varphi: \mathcal{B} \rightarrow \mathcal{A}$ then $\mathcal{A} \otimes_{\mathcal{B}} \mathcal{A}:=$ $\mathcal{A} \otimes_{i m(\varphi)} \mathcal{A}$, and therefore $\operatorname{Tor}^{(\mathcal{A} \mid i m(\varphi))}$ and $\operatorname{Tor}^{(\mathcal{A} \mid \mathcal{B})}$ are one and the same. Then 
since $\varphi$ is r-flat, we have a long exact sequence similar to (2.2) of the form

$$
\cdots \rightarrow \operatorname{Tor}_{p+1}^{(\mathcal{A} \mid \mathcal{B})}(X, Y) \rightarrow \operatorname{Tor}_{p}^{i m(\varphi)}(X, Y) \rightarrow \operatorname{Tor}_{p}^{\mathcal{A}}(X, Y) \rightarrow \operatorname{Tor}_{p}^{(\mathcal{A} \mid \mathcal{B})}(X, Y) \rightarrow \cdots
$$

The result follows since we have isomorphisms of the form

$$
\operatorname{Tor}_{*}^{\mathcal{B}}(X, Y) \cong \operatorname{Tor}_{*}^{i m(\varphi)}(X, Y)
$$

by Proposition 2.1 .

\section{The Jacobi-Zariski sequence for extension groups}

For the time being, we assume $\mathcal{A}$ is a unital $\mathbb{k}$-algebra which is also finite dimensional over $\mathbb{k}$, and $\mathcal{B}$ is an arbitrary unital subalgebra of $\mathcal{A}$. We will work with the category of finitely generated $\mathcal{A}$-modules. In the category of finitely generated $\mathcal{A}$-modules of either parity, every module is necessarily finite dimensional over $\mathbb{k}$. For these categories the $\mathbb{k}$-duality functor $\operatorname{Hom}_{\mathbb{k}}(\cdot, \mathbb{k})$ gives us an equivalence of categories of the form

$$
\operatorname{Hom}_{\mathbb{k}}(\cdot, \mathbb{k}): \mathcal{A}-\bmod \rightarrow \bmod -\mathcal{A},
$$

where $\mathcal{A}$-mod and $\bmod -\mathcal{A}$ denote the categories of finitely generated left and right $\mathcal{A}$ modules, respectively. Using the adjunction between the functors $\otimes_{\mathcal{A}}$ and $\operatorname{Hom}_{\mathbb{k}}(\cdot, \mathbb{k})$, one can easily prove the following lemma.

Lemma 3.1. Assume $\mathcal{A}$ is a finite dimensional $\mathbb{k}$-algebra and let $Y$ be a finitely generated $\mathcal{A}$-module. Then $Y$ is $(\mathcal{A}, \mathcal{B})$-flat if and only if the $\mathbb{k}$-dual $\operatorname{Hom}_{\mathbb{k}}(Y, \mathbb{k})$ is an $(\mathcal{A}, \mathcal{B})$-injective module.

Note that the absolute case is covered by $\mathcal{B}=\mathbb{k}$.

Proof. Assume $Y$ is $(\mathcal{A}, \mathcal{B})$-flat, that is every $(\mathcal{A}, \mathcal{B})$-exact sequence of the form $0 \rightarrow$ $X \rightarrow X^{\prime} \rightarrow X^{\prime \prime} \rightarrow 0$ induce an exact sequence of $\mathbb{k}$-modules the form

$$
0 \rightarrow X \otimes_{\mathcal{A}} Y \rightarrow X^{\prime} \otimes_{\mathcal{A}} Y \rightarrow X^{\prime \prime} \otimes_{\mathcal{A}} Y \rightarrow 0 .
$$

Since $\mathbb{k}$ is a field, and therefore $\operatorname{Hom}_{\mathbb{k}}(\cdot, \mathbb{k})$ is an exact functor, we get another exact sequence of $\mathbb{k}$-modules of the form

$$
0 \rightarrow \operatorname{Hom}_{\mathbb{k}}\left(X \otimes_{\mathcal{A}} Y, \mathbb{k}\right) \rightarrow \operatorname{Hom}_{\mathbb{k}}\left(X^{\prime} \otimes_{\mathcal{A}} Y, \mathbb{k}\right) \rightarrow \operatorname{Hom}_{\mathbb{k}}\left(X^{\prime \prime} \otimes_{\mathcal{A}} Y, \mathbb{k}\right) \rightarrow 0 .
$$

Using the adjunction between $\otimes_{\mathcal{A}}$ and $\operatorname{Hom}_{\mathbb{k}}(\cdot, \mathbb{k})$, we get

$$
\begin{aligned}
0 \rightarrow \operatorname{Hom}_{\mathcal{A}}\left(X, \operatorname{Hom}_{\mathbb{k}}(Y, \mathbb{k})\right) \rightarrow & \operatorname{Hom}_{\mathcal{A}}\left(X^{\prime}, \operatorname{Hom}_{\mathbb{k}}(Y, \mathbb{k})\right) \\
& \rightarrow \operatorname{Hom}_{\mathcal{A}}\left(X^{\prime \prime}, \operatorname{Hom}_{\mathbb{k}}(Y, \mathbb{k})\right) \rightarrow 0
\end{aligned}
$$

is also exact. This is equivalent to the fact that $\operatorname{Hom}_{\mathbb{k}}(Y, \mathbb{k})$ is $(\mathcal{A}, \mathcal{B})$-injective. Conversely, assume $Y$ is $(\mathcal{A}, \mathcal{B})$-injective. Since $\operatorname{Hom}_{\mathbb{k}}(\cdot, \mathbb{k})$ is an equivalence of finitely generated $\mathcal{A}$-modules exchanging parity, there is a finitely generated $\mathcal{A}$-module $T$ of the opposite parity of $Y$ such that $Y \cong \operatorname{Hom}_{\mathbb{k}}(T, \mathbb{k})$. Moreover, one can easily see that $\operatorname{Hom}_{\mathbb{k}}(Y, \mathbb{k}) \cong T$ for the same module since the modules we consider are all finite 
dimensional. Assume now, without any loss of generality, that $Y$ is a right module, and therefore $T$ is left module. Then

$$
\begin{aligned}
0 \rightarrow \operatorname{Hom}_{\mathcal{A}}\left(X, \operatorname{Hom}_{\mathbb{k}}(T, \mathbb{k})\right) \rightarrow & \operatorname{Hom}_{\mathcal{A}}\left(X^{\prime}, \operatorname{Hom}_{\mathbb{k}}(T, \mathbb{k})\right) \\
& \rightarrow \operatorname{Hom}_{\mathcal{A}}\left(X^{\prime \prime}, \operatorname{Hom}_{\mathbb{k}}(T, \mathbb{k})\right) \rightarrow 0
\end{aligned}
$$

is exact for every $(\mathcal{A}, \mathcal{B})$-exact sequence $0 \rightarrow X \rightarrow X^{\prime} \rightarrow X^{\prime \prime} \rightarrow 0$ of finitely generated $\mathcal{A}$-modules. Using the adjunction again we see that

$$
0 \rightarrow \operatorname{Hom}_{\mathbb{k}}\left(X \otimes_{\mathcal{A}} T, \mathbb{k}\right) \rightarrow \operatorname{Hom}_{\mathbb{k}}\left(X^{\prime} \otimes_{\mathcal{A}} T, \mathbb{k}\right) \rightarrow \operatorname{Hom}_{\mathbb{k}}\left(X^{\prime \prime} \otimes_{\mathcal{A}} T, \mathbb{k}\right) \rightarrow 0
$$

is also $(\mathcal{A}, \mathcal{B})$-exact. Since $\operatorname{Hom}_{\mathbb{k}}(\cdot, \mathbb{k})$ is an exact equivalence for finite dimensional $\mathbb{k}$-modules we see that

$$
0 \rightarrow X \otimes_{\mathcal{A}} T \rightarrow X^{\prime} \otimes_{\mathcal{A}} T \rightarrow X^{\prime \prime} \otimes_{\mathcal{A}} T \rightarrow 0
$$

is also $(\mathcal{A}, \mathcal{B})$-exact. This is equivalent to the fact that $T$ is $(\mathcal{A}, \mathcal{B})$-flat.

Lemma 3.2. If $\mathcal{A}$ is a finite dimensional $\mathbb{k}$-algebra and $\mathcal{B} \subseteq \mathcal{A}$ is a $\mathrm{r}$-flat extension, then for any finitely generated $\mathcal{A}$-modules $X$ and $Y$ we have a long exact sequence in cohomology of the form

$$
\cdots \rightarrow \operatorname{Ext}_{(\mathcal{A} \mid \mathcal{B})}^{p}(X, Y) \rightarrow \operatorname{Ext}_{\mathcal{A}}^{p}(X, Y) \rightarrow \operatorname{Ext}_{\mathcal{B}}^{p}(X, Y) \rightarrow \operatorname{Ext}_{(\mathcal{A} \mid \mathcal{B})}^{p+1}(X, Y) \rightarrow \cdots
$$

for every $p \geqslant 0$.

Proof. Assume $X$ and $Y$ are $\mathcal{A}$-modules. Let $\mathcal{U}$ and $\mathcal{V}$ be a unital $\mathbb{k}$-subalgebras of $\mathcal{A}$ such that $\mathcal{V} \subseteq \mathcal{U}$. Fix a pre-dual $T$ of $Y$, i.e., $\operatorname{Hom}_{\mathbb{k}}(T, \mathbb{k}) \cong Y$. Now, observe that if $T$ is left $\mathcal{A}$-module then $\mathrm{CB}_{*}(\mathcal{U} ; \mathcal{U} \mid \mathcal{V} ; T)$ is a $(\mathcal{U}, \mathcal{V})$-projective resolution of $T$, and therefore $\operatorname{Hom}_{\mathbb{k}}\left(\mathrm{CB}_{*}(\mathcal{U} ; \mathcal{U} \mid \mathcal{V} ; T), \mathbb{k}\right)$ is an injective resolution of $Y \cong \operatorname{Hom}_{\mathbb{k}}(T, \mathbb{k})$ by Lemma 3.1. Then

$$
\begin{aligned}
\operatorname{Ext}_{(\mathcal{U} \mid \mathcal{V})}^{n}(X, Y) & \cong \operatorname{Ext}_{(\mathcal{U} \mid \mathcal{V})}^{n}\left(X, \operatorname{Hom}_{\mathbb{k}}(T, \mathbb{k})\right) \\
& =H_{n} \operatorname{Hom}_{\mathcal{U}}\left(X, \operatorname{Hom}_{\mathbb{k}}\left(\operatorname{CB}_{*}(\mathcal{U} ; \mathcal{U} \mid \mathcal{V} ; T), \mathbb{k}\right)\right) \\
& \cong H_{n} \operatorname{Hom}_{\mathbb{k}}\left(\operatorname{CB}_{*}(X ; \mathcal{U} \mid \mathcal{V} ; T), \mathbb{k}\right) \\
& \cong \operatorname{Hom}_{\mathbb{k}}\left(\operatorname{Tor}_{n}^{(\mathcal{U} \mid \mathcal{V})}(X, T), \mathbb{k}\right) .
\end{aligned}
$$

We consider three cases now: (i) $\mathcal{U}=\mathcal{A}$ and $\mathcal{V}=\mathbb{k}$, (ii) $\mathcal{U}=\mathcal{B}$ and $\mathcal{V}=\mathbb{k}$ and finally (iii) $\mathcal{U}=\mathcal{A}$ and $\mathcal{V}=\mathcal{B}$. Then we see that if we apply $\operatorname{Hom}_{\mathbb{k}}(\cdot, \mathbb{k})$ to the long exact sequence $(2.2)$ we obtain the result we would like to prove.

Remark 3.3. Note that in the proof of Lemma 3.1, where we show $\operatorname{Hom}_{\mathbb{k}}(Y, \mathbb{k})$ is $(\mathcal{A}, \mathcal{B})$-injective when it is $(\mathcal{A}, \mathcal{B})$-flat, we did not use the fact that $Y$ is finitely generated over a finite dimensional $\mathbb{k}$-algebra $\mathcal{A}$. Indeed, it is true that for an arbitrary $\mathcal{A}$-module over any $\mathbb{k}$-algebra $\mathcal{A}$ (regardless of $\mathbb{k}$-dimension) and for an arbitrary unital subalgebra $\mathcal{B}$ the module $\operatorname{Hom}_{\mathbb{k}}(Y, \mathbb{k})$ is still $(\mathcal{A}, \mathcal{B})$-injective when $Y$ is $(\mathcal{A}, \mathcal{B})$-flat. Then the result of Lemma 3.2 can be extended to not necessarily finite dimensional algebras, if instead of the full category of finitely generated $\mathcal{A}$-modules we consider the category of $\mathcal{A}$-modules which are finite dimensional over the base field $\mathbb{k}$. The result we proved in Lemma 3.1 in the direction we need is still true and in the category of finite dimensional $\mathcal{A}$-modules, the duality functor is still an equivalence. These are enough to prove an analogue of Lemma 3.2 in the case $\mathcal{A}$ is not necessarily finite dimensional over $\mathbb{k}$. 
Definition 3.4. Assume $\mathcal{A}$ is an arbitrary $\mathbb{k}$-algebra, and we make no assumption on the $\mathbb{k}$-dimension of $\mathcal{A}$. An $\mathcal{A}$-module $M$ is called an approximately finite dimensional $\mathcal{A}$-module if $M$ is a colimit of all of its $\mathcal{A}$-submodules of finite $\mathbb{k}$-dimension.

Theorem 3.5. Let $\mathcal{A}$ be an arbitrary unital $\mathbb{k}$-algebra where we make no assumption on the $\mathbb{k}$-dimension of $\mathcal{A}$. Let $\mathcal{B} \subseteq \mathcal{A}$ be a r-flat extension and assume $Y$ is an approximately finite dimensional $\mathcal{A}$-module. Then for every $\mathcal{A}$-module $X$ we have a long exact sequence of the form (3.1).

Proof. We know by Remark 3.3 that we have a long exact sequence of the form

$$
\cdots \rightarrow \operatorname{Ext}_{(\mathcal{A} \mid \mathcal{B})}^{p}(X, Z) \rightarrow \operatorname{Ext}_{\mathcal{A}}^{p}(X, Z) \rightarrow \operatorname{Ext}_{\mathcal{B}}^{p}(X, Z) \rightarrow \operatorname{Ext}_{(\mathcal{A} \mid \mathcal{B})}^{p+1}(X, Z) \rightarrow \cdots
$$

for every submodule $Z$ of $Y$ of finite $\mathbb{k}$-dimension for every $p \geqslant 0$. Then we use the fact $Y$ is the colimit of all of its $\mathcal{A}$-submodules of finite $\mathbb{k}$-dimension and that colimit is an exact functor.

Corollary 3.6. Assume $\varphi: \mathcal{B} \rightarrow \mathcal{A}$ is a r-flat morphism of unital algebras such that $\operatorname{ker}(\varphi)$ is an $\mathrm{H}$-unital ideal of $\mathcal{B}$. Assume $Y$ is an approximately finite dimensional $\mathcal{A}$ module. Then for every $\mathcal{A}$-module $X$ we have a long exact sequence of the form (3.1).

\section{Jacobi-Zariski sequence for Hochschild homology and cyclic (co)homology}

In this section we assume $\mathcal{A}$ is a unital $\mathbb{k}$-algebra and $\mathcal{B} \subseteq \mathcal{A}$ is a r-flat extension. We make no assumption on the $\mathbb{k}$-dimension of $\mathcal{A}$. Our aim in this section is to repeat the argument we gave in Section 2 and Section 3 for the Hochschild homology and cyclic (co)homology, and prove appropriate versions of Theorem 2.3 and Theorem 3.5. Even though the filtrations are similar, the associated graded complexes will be different. So, we need to check the details carefully.

Now we define an increasing filtration on the Hochschild chain complex by letting

$$
G_{n}^{p}=\sum_{n_{0}+\cdots+n_{p}=n-p} M \otimes \mathcal{B}^{\otimes n_{0}} \otimes \mathcal{A} \otimes \mathcal{B}^{\otimes n_{1}} \otimes \cdots \otimes \mathcal{A} \otimes \mathcal{B}^{\otimes n_{p}}
$$

for $0 \leqslant p \leqslant n$. We let $G_{n}^{p}=0$ for $p<0 \leqslant n$ and $G_{n}^{p}=G_{n}^{p+1}$ for all $p \geqslant n \geqslant 0$. Here again, the filtration degree counts the number of tensor components which are equal to $\mathcal{A}$ and observe that $G_{*}^{0}=\mathrm{CH}_{*}(\mathcal{B}, M)$. Moreover, we see that $\operatorname{colim}_{p} G_{*}^{p}=\mathrm{CH}_{*}(\mathcal{A}, M)$. The associated graded complex is then given by

$$
G_{n}^{p} / G_{n}^{p-1} \cong \bigoplus_{n_{0}+\cdots+n_{p}=n-p} M \otimes \mathcal{B}^{\otimes n_{0}} \otimes(\mathcal{A} / \mathcal{B}) \otimes \mathcal{B}^{\otimes n_{1}} \otimes \cdots \otimes(\mathcal{A} / \mathcal{B}) \otimes \mathcal{B}^{\otimes n_{p}}
$$

for any $n \geqslant p \geqslant 1$, and at degree 0 we see $G_{*}^{0} / G_{*}^{-1}=G_{*}^{0}=\mathrm{CH}_{*}(\mathcal{B}, M)$. But recall that $\mathcal{A} / \mathcal{B}$ is flat over $\mathcal{B}$ since $\mathcal{B} \subseteq \mathcal{A}$ is a r-flat extension. Then on the $E^{1}$-page of the associated spectral sequence we get only two non-zero groups: one on the $p$-axis and the other on the $q$-axis

$$
E_{p, q}^{1}=H_{p+q}\left(G_{*}^{p} / G_{*}^{p-1}\right)= \begin{cases}H H_{q}(\mathcal{B}, M) & \text { if } p=0 \\ \widetilde{C H}_{p}(\mathcal{A} \mid \mathcal{B}, M) & \text { if } q=0 \text { and } p>0,\end{cases}
$$

where $\widetilde{C H}_{*}(\mathcal{A} \mid \mathcal{B}, M)$ is the normalized relative Hochschild complex $[\mathbf{8}, 1.1 .14]$. This 
leads us to the $E^{2}$-page

$$
E_{p, q}^{2}= \begin{cases}H H_{q}(\mathcal{B}, M) & \text { if } p=0 \text { and } q>0 \\ H H_{p}(\mathcal{A} \mid \mathcal{B}, M) & \text { if } q=0\end{cases}
$$

The rest of the argument is similar to the argument we gave in Section 2.

Theorem 4.1. Assume $\mathcal{B} \subseteq \mathcal{A}$ is a $\mathrm{r}$-flat extension of unital, associative but not necessarily commutative $\mathbb{k}$-algebras. Then for any $\mathcal{A}$-bimodule $M$ we have a long exact sequence of the form

$$
\cdots \rightarrow H H_{p}(\mathcal{B}, M) \rightarrow H H_{p}(\mathcal{A}, M) \rightarrow H H_{p}(\mathcal{A} \mid \mathcal{B}, M) \rightarrow H H_{p-1}(\mathcal{B}, M) \rightarrow \cdots
$$

for $p \geqslant 0$. If we assume $M$ is approximately finite dimensional, then we obtain a long exact sequence in cohomology of the form

$$
\cdots \rightarrow H H^{p}(\mathcal{A} \mid \mathcal{B}, M) \rightarrow H H^{p}(\mathcal{A}, M) \rightarrow H H^{p}(\mathcal{B}, M) \rightarrow H H^{p+1}(\mathcal{A} \mid \mathcal{B}, M) \rightarrow \cdots
$$

for every $p \geqslant 0$.

Now we are ready to extend this result to cyclic (co)homology.

Theorem 4.2. Assume $\mathcal{A}, \mathcal{B}$ and $\mathbb{k}$ are as before. Then we have the following long exact sequences:

$$
\begin{aligned}
\cdots \rightarrow H H_{p+1}(\mathcal{A} \mid \mathcal{B}) & \rightarrow H H_{p}(\mathcal{B}) \rightarrow H H_{p}(\mathcal{A}) \rightarrow H H_{p}(\mathcal{A} \mid \mathcal{B}) \rightarrow \cdots \\
\cdots \rightarrow H C_{p+1}(\mathcal{A} \mid \mathcal{B}) & \rightarrow H C_{p}(\mathcal{B}) \rightarrow H C_{p}(\mathcal{A}) \rightarrow H C_{p}(\mathcal{A} \mid \mathcal{B}) \rightarrow \cdots \\
\cdots & \rightarrow H C^{p}(\mathcal{A} \mid \mathcal{B}) \rightarrow H C^{p}(\mathcal{A}) \rightarrow H C^{p}(\mathcal{B}) \rightarrow H C^{p+1}(\mathcal{A} \mid \mathcal{B}) \rightarrow \cdots
\end{aligned}
$$

Proof. In order to prove our assertion for cyclic (co)homology, we first need to derive (4.2) which is a version of the Jacobi-Zariski sequence for Hochschild homology. For this purpose, we will need to write a filtration similar to the filtration we defined in (4.1). So, for $0 \leqslant p \leqslant n+1$ we define

$$
G_{n}^{p}=\sum_{n_{0}+\cdots+n_{p}=n+1-p} \mathcal{B}^{\otimes n_{0}} \otimes \mathcal{A} \otimes \mathcal{B}^{\otimes n_{1}} \otimes \cdots \otimes \mathcal{A} \otimes \mathcal{B}^{\otimes n_{p}} .
$$

We let $G_{n}^{p}=G_{n}^{p+1}$ for all $p \geqslant n+1$ and $G_{n}^{p}=0$ for $p<0$. Observe that the filtration is compatible with the actions of the cyclic groups $\mathbb{Z} /(n+1)$ at every degree $n \geqslant 0$. The rest of the proof for obtaining the long exact sequence (4.2) is similar to argument we used at the beginning of this section for Hochschild homology with coefficients and the proof of Theorem 2.3. The proof for cyclic (co)homology also follows after our observation that the filtration $G_{*}^{p}$ is compatible with the actions of the cyclic groups.

Theorem 4.3. Let $\varphi: \mathcal{B} \rightarrow \mathcal{A}$ be a $\mathrm{r}$-flat morphism of unital $\mathbb{k}$-algebras such that $\mathcal{I}:=\operatorname{ker}(\varphi)$ is $\mathrm{H}$-unital. Let $\mathrm{CH}_{*}(\mathcal{A}, \mathcal{B})$ be the homotopy cofiber of the morphism $\varphi_{*}: \mathrm{CH}_{*}(\mathcal{B}) \rightarrow \mathrm{CH}_{*}(\mathcal{A})$. Then there is a homotopy cofibration sequence of the form

$$
\Sigma \mathrm{CH}_{*}(\mathcal{I}) \rightarrow \mathrm{CH}_{*}(\mathcal{A}, \mathcal{B}) \rightarrow \mathrm{CH}_{*}(\mathcal{A} \mid \mathcal{B})
$$

which induces a long exact sequence of the form

$$
\cdots \rightarrow H H_{p+1}(\mathcal{A} \mid \mathcal{B}) \rightarrow H H_{p-1}(\mathcal{I}) \rightarrow H H_{p}(\mathcal{A}, \mathcal{B}) \rightarrow H H_{p}(\mathcal{A} \mid \mathcal{B}) \rightarrow \cdots
$$

for $p \geqslant 0$. Analogous sequences exist for the cyclic homology and cohomology with no additional hypothesis. 
Proof. We will give the proof for the Hochschild homology. The proofs for cyclic homology and cohomology are similar, and therefore, omitted. We consider all of our Hochschild chain complexes as differential graded $\mathbb{k}$-modules inside (bounded below) derived category of $\mathbb{k}$-modules. This is a triangulated category. In this category we have two distinguished triangles: one coming from the Wodzicki excision sequence $[\mathbf{1 3}$, Thm. 3.1], and the other coming from our Jacobi-Zariski sequence:

$$
\mathrm{CH}_{*}(\mathcal{I}) \rightarrow \mathrm{CH}_{*}(\mathcal{B}) \stackrel{\pi_{*}}{\longrightarrow} \mathrm{CH}_{*}(\mathcal{B} / \mathcal{I}) \rightarrow \Sigma \mathrm{CH}_{*}(\mathcal{I})
$$

and

$$
\mathrm{CH}_{*}(\mathcal{B} / \mathcal{I}) \stackrel{i_{*}}{\longrightarrow} \mathrm{CH}_{*}(\mathcal{A}) \rightarrow \mathrm{CH}_{*}(\mathcal{A} \mid \mathcal{B}) \rightarrow \Sigma \mathrm{CH}_{*}(\mathcal{B} / \mathcal{I}) .
$$

Now consider another homotopy cofibration sequence

$$
\mathrm{CH}_{*}(\mathcal{B}) \stackrel{\varphi_{*}}{\longrightarrow} \mathrm{CH}_{*}(\mathcal{A}) \rightarrow \mathrm{CH}_{*}(\mathcal{A}, \mathcal{B}),
$$

where the last term is defined as the homotopy cofiber of the morphism $\varphi_{*}: \mathrm{CH}_{*}(\mathcal{B}) \rightarrow$ $\mathrm{CH}_{*}(\mathcal{A})$. We construct

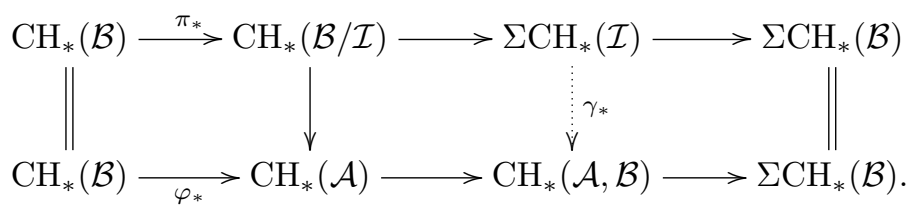

Now, $\gamma_{*}$ exists because the (bounded below) derived category of $\mathbb{k}$-modules is a triangulated category. We also have that the middle square is homotopy cartesian $[\mathbf{1 1}$, Lem. 1.4.3]. Moreover, we have a distinguished triangle of the form

$$
\Sigma \mathrm{CH}_{*}(\mathcal{I}) \stackrel{\gamma_{*}}{\longrightarrow} \mathrm{CH}_{*}(\mathcal{A}, \mathcal{B}) \rightarrow \mathrm{CH}_{*}(\mathcal{A} \mid \mathcal{B}) \rightarrow \Sigma^{2} \mathrm{CH}_{*}(\mathcal{I})
$$

since $\mathrm{CH}_{*}(\mathcal{A} \mid \mathcal{B})$ is the homotopy cofiber of the morphism $\mathrm{CH}_{*}(\mathcal{B} / \mathcal{I}) \rightarrow \mathrm{CH}_{*}(\mathcal{A})$ induced by the inclusion $\operatorname{im}(\varphi) \subseteq \mathcal{A}$ because of Theorem 4.2. This finishes the proof.

One can easily see that Theorem 4.3 gives us the Jacobi-Zariski sequence when $\varphi$ is monomorphism with an r-flat image and the Wodzicki excision sequence when $\varphi$ is an epimorphism with an H-unital kernel, for Hochschild homology and cyclic (co)homology.

Conjecture 4.4. We believe that an appropriate analogue of Theorem 4.2 holds for Hochschild cohomology when $p$ is large enough. However, due to its non-functoriality, to prove such an analogue for Hochschild cohomology is not a straightforward task. If $\mathcal{B} \subseteq \mathcal{A}$ is an arbitrary extension, then we have a homotopy cofibration sequence of the form $\mathrm{CH}^{*}(\mathcal{B}) \rightarrow \mathrm{CH}^{*}(\mathcal{B}, \mathcal{A}) \rightarrow \mathrm{CH}^{*}(\mathcal{B}, \mathcal{A} / \mathcal{B})$ coming from the short exact sequence of $\mathcal{B}$-bimodules $0 \rightarrow \mathcal{B} \rightarrow \mathcal{A} \rightarrow \mathcal{A} / \mathcal{B} \rightarrow 0$. The last term $\operatorname{CH}^{*}(\mathcal{B}, \mathcal{A} / \mathcal{B})$ calculates $H H^{*}(\mathcal{B}, \mathcal{A} / \mathcal{B})$, which is $\operatorname{Ext}_{\mathcal{B}^{e}}^{*}(\mathcal{B}, \mathcal{A} / \mathcal{B})$. So, we have $H H^{n}(\mathcal{B}) \cong H H^{n}(\mathcal{B}, \mathcal{A})$ for $n \geqslant 2$ when $\mathcal{A} / \mathcal{B}$ is an injective $\mathcal{B}^{e}$-module. If $\mathcal{A}$ is approximately finite dimensional and $\mathcal{A} / \mathcal{B}$ is $\mathcal{B}^{e}$-flat on top of being $\mathcal{B}^{e}$-injective, then we can use Theorem 4.1 where we set $M=\mathcal{A}$ to obtain an analogue of Theorem 4.2 for Hochschild cohomology but for $p \geqslant 2$. There are similar results pointing in this direction. See, for example, [1, Thm. 4.5], [5, Thm. 5.3], and [10]. 


\section{References}

[1] C. Cibils, Tensor Hochschild homology and cohomology, in Interactions between ring theory and representations of algebras (Murcia), Lecture Notes in Pure and Appl. Math. 210, pages 35-51, Dekker, New York, 2000.

[2] J.A. de la Peña and C. Xi, Hochschild cohomology of algebras with homological ideals, Tsukuba J. Math. 30 (2006), no. 1, 61-79.

[3] W. Geigle and H. Lenzing, Perpendicular categories with applications to representations and sheaves, J. Algebra 144 (1991), no. 2, 273-343.

[4] M. Gerstenhaber and S.D. Schack, Relative Hochschild cohomology, rigid algebras, and the Bockstein, J. Pure Appl. Algebra 43 (1986), no. 1, 53-74.

[5] D. Happel, Hochschild cohomology of finite-dimensional algebras, Lecture Notes in Mathematics 1404 (1989), 108-126.

[6] G. Hochschild, Relative homological algebra, Trans. Amer. Math. Soc. 82 (1956), no. 1, 246-269.

[7] A. Kaygun, Uniqueness of pairings in Hopf-cyclic cohomology, Journal of K-theory 6 (2010), no. 1, 1-21.

[8] J.-L. Loday, Cyclic homology, Die Grundlehren der Mathematischen Wissenschaften 301, Springer-Verlag, New York, second edition, 1998.

[9] S. MacLane, Homology, Die Grundlehren der mathematischen Wissenschaften 114, Academic Press Inc., New York, 1963.

[10] S. Michelena and M.I. Platzeck, Hochschild cohomology of triangular matrix algebras, J. Algebra 233 (2000), no. 2, 502-525.

[11] A. Neeman, Triangulated categories, Annals of Mathematics Studies 148, Princeton University Press, Princeton, N.J., 2001.

[12] D. Quillen, On the (co-) homology of commutative rings, in Applications of categorical algebra (Proc. Sympos. Pure Math., Vol. XVII, New York, 1968), pages 65-87, Amer. Math. Soc., Providence, R.I., 1970.

[13] M. Wodzicki, Excision in cyclic homology and in rational algebraic $K$-theory, Ann. of Math. 129 (1989), no. 3, 591-639.

Atabey Kaygun atabey.kaygun@bahcesehir.edu.tr

Department of Mathematics and Computer Science, Bahçeşehir University, Çrağan Cad. Beşiktaş, Istanbul 34359, Turkey 